\title{
PROCEDIMIENTO PARA ESTIMAR LA DIVERSIDAD ESPACIAL EN AMBIENTES ELECTROMAGNÉTICOS DE MULTITRAYECTORIAS
}

\section{PROCEDURE TO ESTIMATE THE SPATIAL DIVERSITY IN ELECTROMAGNETIC ENVIRONMENTS OF MULTIPATHS}

\author{
J.H. Caltenco $^{1} \quad$ R. Linares y Miranda ${ }^{1} \quad$ J. López-Bonilla $^{1} \quad$ C. Sosa-Caraveo $^{1}$ \\ Recibido 17 de junio de 2008, aceptado 18 de junio de 2009 \\ Received: June 17, 2008 Accepted: June 18, 2009
}

\begin{abstract}
RESUMEN
En este trabajo se presenta un procedimiento para estimar la diversidad espacial en ambientes electromagnéticos de multitrayectorias, utilizando una antena de dos elementos (combinación dipolo aro-capacitor). La diversidad espacial se determina a partir del ángulo de incidencia de las señales electromagnéticas que llegan a la antena y de los coeficientes de correlación. El ángulo de incidencia permite también identificar las componentes de campo electromagnético, lo que muestra que la antena de dos elementos es una herramienta útil para el análisis de multitrayectorias, lo cual se prueba experimentalmente.
\end{abstract}

Palabras clave: Multitrayectorias, diversidad espacial, ángulo de incidencia.

\begin{abstract}
This paper presents a procedure to estimate the spatial diversity in electromagnetic environments of multipaths, using an antenna of two elements (combination dipole hoop-capacitor). The spatial diversity is determined from the angle of incidence of electromagnetic signals arriving at the antenna and from correlation coefficients. This angle also allows to identify the components of the electromagnetic field, which shows that the two elements antenna is a useful tool to analyze multipaths, which is tested experimentally.
\end{abstract}

Keywords: Multipaths, spatial diversity, angle of incidence.

\section{INTRODUCCIÓN}

Los sistemas de telecomunicaciones mantienen su importancia en el desarrollo de la industria y los negocios. En este contexto, las comunicaciones inalámbricas personales han alcanzado una conectividad sin precedentes en cualquier parte del mundo. A pesar de esto, el acceso inalámbrico tiene un inevitable compromiso con el uso de un espectro de radio finito, el cual presenta un ambiente de multitrayectorias. Por consiguiente, el desafío que enfrentan los ingenieros de comunicaciones es el desarrollo de modelos útiles para predecir el comportamiento de los sistemas inalámbricos con la mayor exactitud y eficiencia posible. Estos sistemas se han desarrollado para satisfacer la incesante demanda de los nuevos servicios de banda ancha y su fiabilidad funcional dentro de espacios abiertos y cerrados. Sin embargo, aún no se ha alcanzado un alto porcentaje de fiabilidad. El diseño y funcionamiento de dichos sistemas son evidentemente determinados por la interacción entre la antena y las propiedades del ambiente electromagnético. Por lo tanto, para analizar, diseñar e implementar apropiadamente tales sistemas se deberá tener un conocimiento fiable de los mecanismos físicos de propagación del ambiente electromagnético.

En las ciudades con alta densidad de edificios, el ambiente electromagnético es complejo, porque las ondas electromagnéticas encuentran múltiples obstáculos y sufren dispersiones, reflexiones y difracciones, generando así un ambiente de multitrayectorias. Una antena receptora operando en dicho ambiente recibe varias ondas electromagnéticas y la información útil se encuentra

\footnotetext{
1 Instituto Politécnico Nacional. Escuela Superior de Ingeniería Mecánica y Eléctrica. Sección de Estudios de Postgrado e Investigación. Edif. Z-4, 3er Piso Col. Lindavista CP 07738. México D.F. E-mail: jlopezb@ipn.mx
} 
degenerada. Se conoce ampliamente que las antenas inteligentes mejoran el comportamiento de los enlaces de radio en ambientes de multitrayectorias y que la diversidad espacial se puede compartir entre el transmisor y el receptor. Además, dichas antenas radian y reciben energía en una dirección prefijada, por lo que algunos efectos del ambiente de multitrayectorias se reducen. En el tópico de antenas adaptivas hay varias publicaciones [1-4] motivando el diseño de arreglos basados en sistemas; sin embargo, aún existen muchas preguntas alrededor de dichos sistemas, algunas de estas son: ¿Cómo se debe de configurar una antena?, ¿Qué tipo de procesamiento de señal cubrirá los accesos y usuarios múltiples?, ¿Cómo se debe codificar la señal para hacer eficiente el uso del arreglo de antenas?, y ¿Cómo puede evaluarse el desempeño? Hay diferentes compromisos dentro de los sistemas inalámbricos y aún no se ha encontrado una respuesta completa para esta problemática. Es evidente la necesidad de conocer los efectos de degradación causados por el canal de propagación antes de dar cualquier respuesta a las preguntas anteriores.

Un modelado teórico no es suficiente para analizar los ambientes de propagación, pero tampoco lo es una descripción empírica basada en mediciones, ya que es difícil identificar las características de propagación basadas solamente en los datos medidos. Las mediciones realizadas en ambientes típicos cerrados y abiertos presentan superposición de los diferentes mecanismos de propagación de multitrayectorias, debido a dispersiones, difracciones y reflexiones que son difíciles de identificar por separado. Las técnicas de diversidad espacial se han desarrollado como un método efectivo para minimizar los problemas de desvanecimiento rápido en ambientes de multitrayectorias [5-7], cuyas características se han explorado usando modelos teóricos, que de acuerdo con los resultados encontrados la diversidad espacial presenta beneficios significativos; por ejemplo: en [6] se especifica que en un arreglo de antenas el espacio entre elementos de $0.75 \lambda$ a $1 \lambda$ es suficiente para alcanzar un funcionamiento óptimo.

El ángulo de incidencia (ADI) es un parámetro de conectividad que permite la estimación del tiempo de incidencia (TDI) y la diferencia de tiempos de incidencia (DTDI). Estos son importantes en la localización de sistemas y dan información que ayuda a analizar los problemas de multitrayectorias. El análisis de multitrayectorias en base a ADI y TDI se aplica en diversas áreas urbanas donde es particularmente difícil la propagación de ondas electro-magnéticas, debido a la formación de ductos, tanto en el exterior de edificios como en el interior de ellos, que provocan la reflexión y difracción de las señales.
Hay diferentes técnicas para estimar el ADI, el TDI y la DTDI, y en general todas ellas se han desarrollado con antenas adaptivas, las cuales explotan el análisis tiempoespacio y suponen que se conoce a priori la forma del pulso transmitido [7-8].

Tradicionalmente, las antenas de aro se han usado para encontrar la dirección de la señal de incidencia. Esta aplicación se da cuando el plano del aro se orienta verticalmente y la señal incidente tiene una polarización vertical y entonces la antena se gira. La medición de referencia son los nulos y los máximos de la respuesta de la antena, pero esta práctica tiene dos ambigüedades para encontrar la dirección de incidencia (DOI), debido a la existencia de nulos en ambos lados del patrón de radiación de la antena de aro. Esta ambigüedad se ha resuelto con la combinación de una antena de aro y una antena dipolo, las cuales se diseñan para que las amplitudes de las respuestas sean iguales y así producen un patrón cardioide que tiene un nulo y un máximo. Esta antena se gira para determinar la dirección de la señal incidente.

Una antena con la misma idea, pero optimizada respecto a los nulos, es un dipolo terminado con una combinación en paralelo de antena de aro y capacitor. Esta antena explota el conocimiento de fase de la corriente entre el dipolo y el aro que produce el capacitor. En ambientes electromagnéticos de multitrayectorias, donde los parámetros son de naturaleza aleatoria, esta antena reduce la probabilidad de profundidad de los nulos, independientemente de la polarización de la señal incidente y del ángulo con que incide. Los resultados se analizan desde el punto de vista probabilístico, usando un modelo que considera solo unas cuantas trayectorias dominantes y suponiendo que se conoce la relación entre las señales incidentes y reflejadas, por lo que se usa el concepto de trayectoria dominante a partir de los resultados probabilísticos. Este procedimiento no es útil para determinar la dirección de la señal incidente, pero la antena, por su simplicidad y la ventaja de reducción de la profundidad de los nulos, puede utilizarse con otro procedimiento para analizar el ambiente electromagnético de multitrayectorias.

En un esfuerzo por conocer y analizar los mecanismos de propagación del ambiente de multitrayectorias se han propuesto diferentes antenas [10-14], que en general todas ellas utilizan métodos complejos de adquisición de datos. Con este objetivo en [15-16] se presenta un procedimiento para analizar señales de multitrayectorias de acuerdo con la amplitud de los componentes de la señal y los ángulos de incidencia. En este caso se aplica el concepto de patrón de interferencia espacial dominante y se muestra una 
aplicación para un enlace de radio en espacios abiertos de una zona urbana.

En este trabajo se presenta el análisis de un ambiente de multitrayectorias utilizando una antena dipolo terminada con una combinación en paralelo de antena de aro y capacitor. También se aplica el concepto de patrón de interferencia espacial dominante y el concepto de trayectoria dominante. El ángulo de incidencia de la señal se obtiene por medio de la Transformada Discreta de Fourier (TDF) para diferentes coeficientes de reflexión y se desarrolla un coeficiente de correlación para determinar la distancia óptima entre dos antenas o la distancia entre antena y plano de reflexión para un mínimo de profundidad de nulos. Técnicas similares basadas en el análisis de un patrón de distribución de amplitud vertical se han usado para el cálculo de coeficiente de reflexión de tierra [17]. Con el mismo propósito en [18] se usó el desplazamiento horizontal de un receptor. En general, las publicaciones respecto al ángulo de incidencia y tiempos de incidencia de la señal en una antena, reportan resultados con técnicas complejas de procesamiento de información. En nuestro caso el análisis se hace directo utilizando instrumentación estándar de laboratorio, ya que la antena de dos elementos es simple y bastante eficiente. Por consiguiente, esta técnica puede aplicarse a casos generales de trayectorias de señales que forman el ambiente multitrayectorias. Los resultados se presentan para condiciones reales que se pueden encontrar en ambientes cerrados y abiertos a través de los procesos de simulación.

Este trabajo tiene la siguiente distribución: en la siguiente sección se presenta el escenario de propagación para mostrar las ventajas de la antena de dos elementos y la disminución de nulos cuando se anexa el capacitor, así como también se analiza el comportamiento de la antena en presencia de un plano reflector no ideal, considerando el ángulo de inclinación y de polarización. A continuación, se da el procedimiento para determinar el ángulo de incidencia de la señal que se recibe en una antena y la obtención de un patrón para la medición de dichos ángulos. En la sección "Diversidad espacial", se determinan los coeficientes de correlación que identifican la diversidad espacial respecto a las trayectorias dominantes. Finalmente, se reportan los datos de la parte experimental que comprueba los modelos desarrollados, y desde luego también se presentan las conclusiones y referencias.

\section{ESCENARIO DE PROPAGACIÓN}

El comportamiento de la antena que se usa se describe utilizando el escenario mostrado en la figura 1 , en donde se varía la polarización de la onda incidente y los coeficientes de reflexión del plano reflector.

Bajo condiciones ideales las características de radiación de una antena pueden considerarse dentro de un medio sin límites. Pero la presencia de obstáculos, especialmente cuando están cerca de la antena, puede alterar significativamente todas las propiedades de radiación del sistema de la antena. En la práctica, una antena receptora es frecuentemente afectada por campos electromagnéticos indeseables que provienen de la interacción de los modos de propagación causados por efectos de difracción y reflexión y otros procesos que dificultan la transmisión. Todos los problemas mencionados en primera instancia se presentan como nulos en el patrón de radiación y se han publicado varios trabajos al respecto [10-14], los cuales muestran metodologías para eliminar o minimizar el problema de los nulos. Por ejemplo, en [14] una pequeña antena de aro compuesta por dos placas de cobre paralelas se cortocircuitan en diferentes puntos para modificar el patrón de radiación y eliminar los nulos. Los cortocircuitos se mueven por medio de electrónica asociada a la antena y de esta forma se mejora la polarización de la antena. Los efectos de polarización también se observan en la antena que se utiliza en este trabajo (antena de dos elementos).

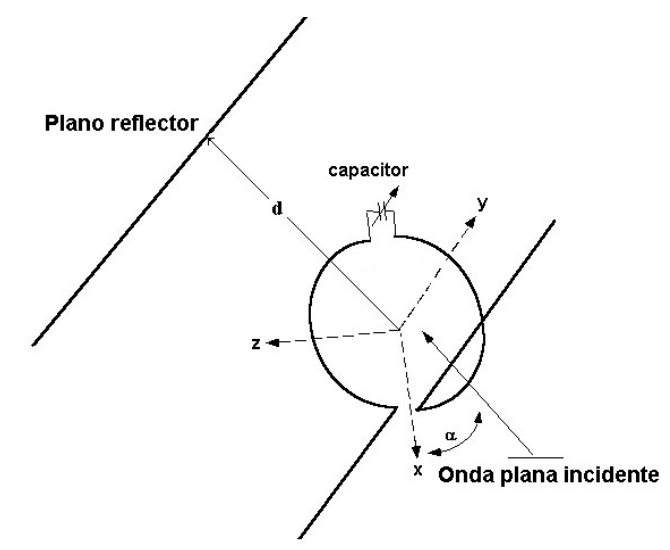

Figura 1. Geometría del escenario de propagación.

En nuestro caso los nulos del patrón de radiación también son minimizados utilizando la antena de dos elementos, cuya ventaja de la combinación antena de aro-capacitor se presentan para una onda plana incidente con ángulo de polarización de $0^{\circ}, 45^{\circ}$ y $90^{\circ}$. Estos efectos se ilustran en las figuras 2 y 3 con relación al patrón de polarización sin capacitor. 


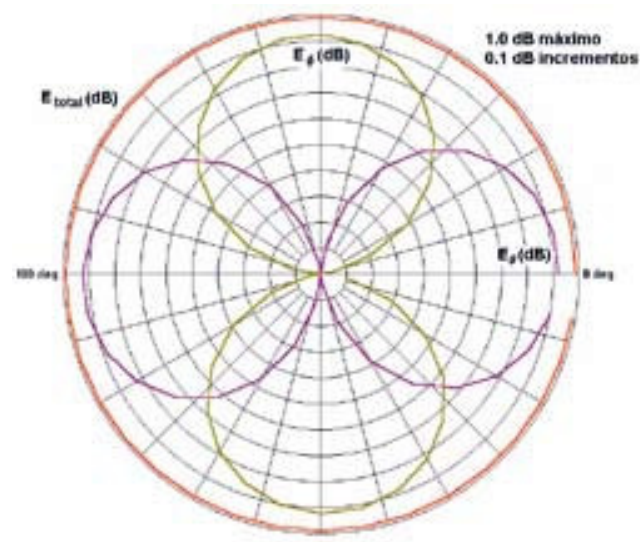

Figura 2a. Patrones relativos de campo eléctrico de una antena de dos elementos, antena dipolo-aro.

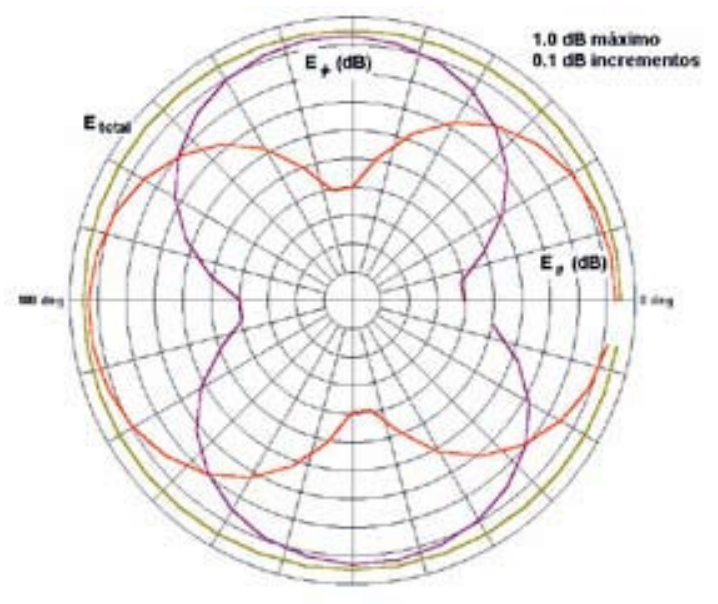

Figura 2b. Patrones relativos de campo eléctrico de una antena de dos elementos, antena dipolo-arocapacitor.

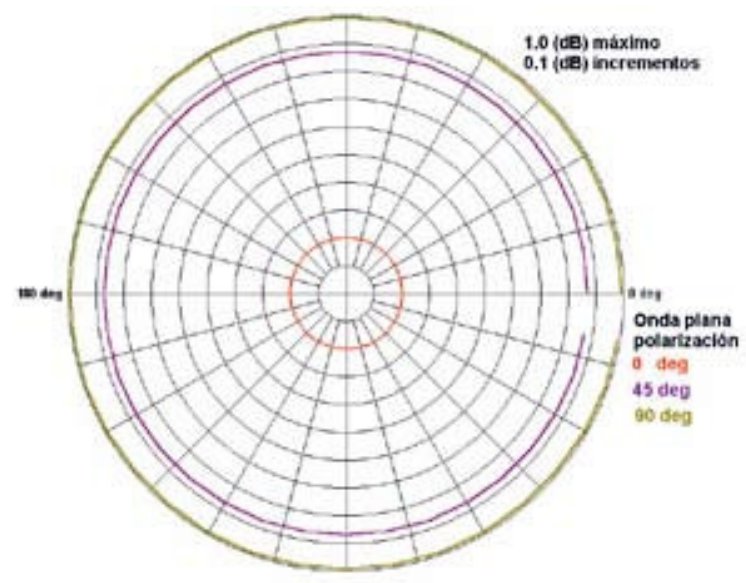

Figura 3a. Patrones relativos del campo eléctrico total de una antena de dos elementos para diferentes polarizaciones de una onda plana incidente, antena dipolo-aro.

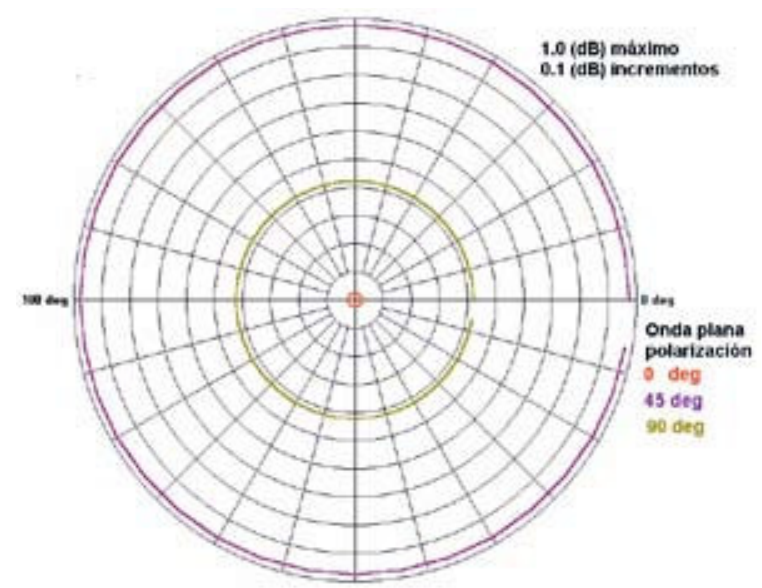

Figura 3b. Patrones relativos del campo eléctrico total de una antena de dos elementos para diferentes polarizaciones de una onda plana incidente, antena dipolo-aro-capacitor.

\section{Comportamiento de los coeficientes de reflexión}

En un ambiente de multitrayectorias la antena de dos elementos responde a las ondas directas y reflejadas $\left(E_{a}\right.$ y $\left.H_{a}\right)$. Cuando la antena dipolo se alinea con su eje-a, el voltaje de salida de la antena de dos elementos puede determinarse por

$$
V=C\left|E_{a}+\eta_{o} H_{a} e^{j(\gamma+\pi / 2)}\right|
$$

donde $\mathrm{C}$ es una ganancia constante que tiene unidades de longitud, $\eta_{o}$ es la impedancia del espacio libre, $\gamma$ es la fase extra debido al capacitor que se introduce entre la antena dipolo y de aro, y $H_{a}=E_{a} /$ y $\eta_{o}$.

Para la onda directa y reflejada incidiendo en la antena de dos elementos, las ecuaciones de las componentes del campo son:

$$
\begin{aligned}
& E_{a}=E_{z}=\cos \Psi\left(E_{\theta}^{i} e^{j \varphi}+\Gamma_{\perp} E_{\theta}^{r} e^{-j \varphi}\right) \\
& H_{a}=H_{z}=\sin \Psi\left(H_{\phi}^{i} e^{j \varphi}+\Gamma_{\|} H_{\phi}^{r} e^{-j \varphi}\right)
\end{aligned}
$$

donde $E_{\theta}^{i}, H_{\phi}^{i}$ y $E_{\theta}^{r}, H_{\phi}^{r}$ son los coeficientes de ganancia compleja de campo lejano de la trayectoria directa y reflejada. $\psi$ es el ángulo de polarización. $\varphi=k_{o} d \cos \alpha$, $k_{o}$ es la constante de propagación, $d$ es la distancia entre la antena y el plano reflector, y $\alpha$ es el ángulo de incidencia. $\Gamma_{\perp}$ y $\Gamma_{\|}$corresponden a los coeficientes de reflexión 
para el campo eléctrico perpendicular y paralelo al plano creado por el vector de propagación normal a la superficie y pueden determinarse por

$$
\begin{gathered}
\left.\Gamma_{\perp}\right|_{\mu_{1}=\mu_{2}}=\frac{\cos \vartheta_{i}-\sqrt{\mathrm{k}-\sqrt{\mathrm{k}} \sin ^{2} \vartheta_{i}}}{\cos \vartheta_{i}+\sqrt{\mathrm{k}-\sqrt{\mathrm{k}} \sin ^{2} \vartheta_{i}}}, \\
\Gamma_{\|\left.\right|_{\mu_{1}=\mu_{2}}=}=\frac{-\cos \vartheta_{i}+\sqrt{\mathrm{k}-\sqrt{\mathrm{k}} \sin ^{2} \vartheta_{i}}}{\cos \vartheta_{i}+\sqrt{\mathrm{k}-\sqrt{\mathrm{k}} \sin ^{2} \vartheta_{i}}},
\end{gathered}
$$

donde $\vartheta_{i}$ es el ángulo de incidencia al plano reflector, $\mathrm{k}$ es la constante dieléctrica del plano reflector.

Sustituyendo las componentes de los campos en la ec. (1) se obtiene lo siguiente

$$
\begin{aligned}
V_{\text {out }}=C \mid\{ & \left.(\cos \Psi)+(\sin \Psi) e^{j(\gamma+\pi / 2)}\right\} E_{\theta}^{i} e^{-j \varphi}+ \\
& \left\{\left(\Gamma_{\perp} \cos \Psi\right)+\left(\Gamma_{\|} \sin \Psi\right) e^{j(\gamma+\pi / 2)}\right\} E_{\theta}^{r} e^{j \varphi} \mid
\end{aligned}
$$

En la ec. (6), el primer término es máximo cuando $\Psi=45^{\circ}$ (figura $3 \mathrm{~b}$ ), sin embargo, el segundo término depende de los coeficientes de reflexión, si este se analiza $\left(\Gamma_{\perp} \cos \Psi+\Gamma_{\|} \sin \Psi\right)$ para diferentes constantes dieléctricas y ángulos de polarización se pueden determinar límites donde los efectos del plano de reflexión son más críticos para la generación de nulos. El comportamiento de este término se muestra en la figura 4.

De la figura 4 , se puede concluir que las trayectorias dominantes se presentan en el intervalo $0^{\circ}$ a $45^{\circ}$, tanto para el ángulo de incidencia respecto al plano reflector, como para el ángulo de polarización. En este intervalo los niveles de amplitud del voltaje de salida de la antena son bajos para constantes dieléctricas altas. Para constantes dieléctricas bajas, $\Psi>45^{\circ}$ (ángulo de polarización) y $\vartheta>45^{\circ}$ (ángulo de incidencia al plano reflector), los niveles de amplitud de la señal de salida de la antena tienen variaciones. Con respecto a la figura 4 , las variaciones son $\pm 6 d B$.

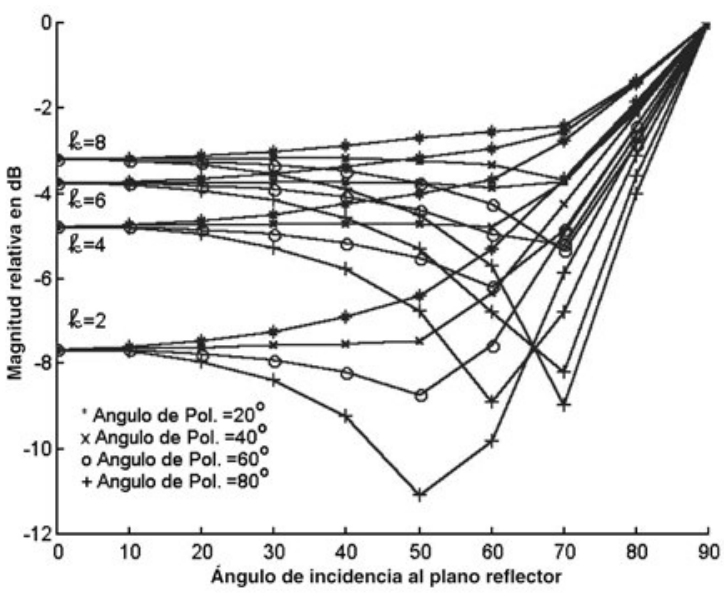

Figura 4. Comportamiento del segundo término de la ecuación (6) con relación al ángulo de polarización y al ángulo de incidencia del plano reflector y los coeficientes de reflexión.

\section{Análisis de polarización e inclinación}

Bajo la suposición de una onda plana incidente desde un plano idealmente reflector colocado en el plano $x-y$, los componentes del campo $\left(E_{a}\right.$ y $\left.H_{a}\right)$ a lo largo del eje- $a$ son

$$
\begin{gathered}
E_{a}=-2 j E_{o} \cos (\psi) \cos \left(\theta_{a}\right) \sin (\varphi) \\
H_{a}=-2\left(E_{o} / \eta_{o}\right) \sin (\psi) \sin \left(\theta_{a}\right) \cos (\varphi)
\end{gathered}
$$

donde $\Psi$ es el ángulo de polarización, $\theta_{a}$ es el ángulo de inclinación, y $\varphi=k_{o} d \cos \alpha$.

Se supone que la onda electromagnética es estacionaria, y se omite la dependencia del tiempo $\exp (j \omega t-\zeta)$ que puede tener una fase arbitraria.

Sustituyendo (7) y (8) en la ec. (1) del voltaje, se tiene

$$
V_{o}=C\left|T_{1}+j\left(T_{2 A}+T_{2 B}\right)\right|
$$

donde

$$
\begin{aligned}
& T_{1}=-2 E_{o} \sin (\psi) \sin \left(\theta_{a}\right) \cos (\varphi) \cos (\gamma+\pi / 2) \\
& T_{2 A}=-2 E_{o} \sin (\psi) \sin \left(\theta_{a}\right) \cos (\varphi) \sin (\gamma+\pi / 2) \\
& T_{2 B}=-2 E_{o} \cos (\psi) \cos \left(\theta_{a}\right) \sin (\varphi) .
\end{aligned}
$$


Los términos $T_{1}$ y $T_{2 A}$ corresponden al campo magnético y son afectados por la fase extra que introduce el capacitor, y $T_{2 B}$ corresponde al campo eléctrico. Graficando la expresión del voltaje para diferentes ángulos de polarización y de inclinación se puede analizar el comportamiento respecto a la generación de nulos. Para una señal incidiendo con un ángulo de $45^{\circ}$ las gráficas del voltaje se muestran en las figuras 5 a 9 .

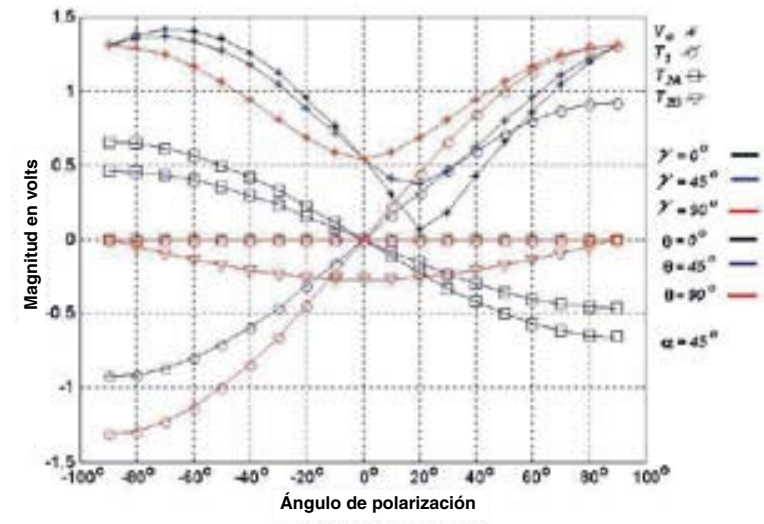

Figura 5. Efectos del ángulo de polarización para diferentes ángulos de inclinación, considerando el corrimiento de fase que puede o no producir el capacitor en la antena.

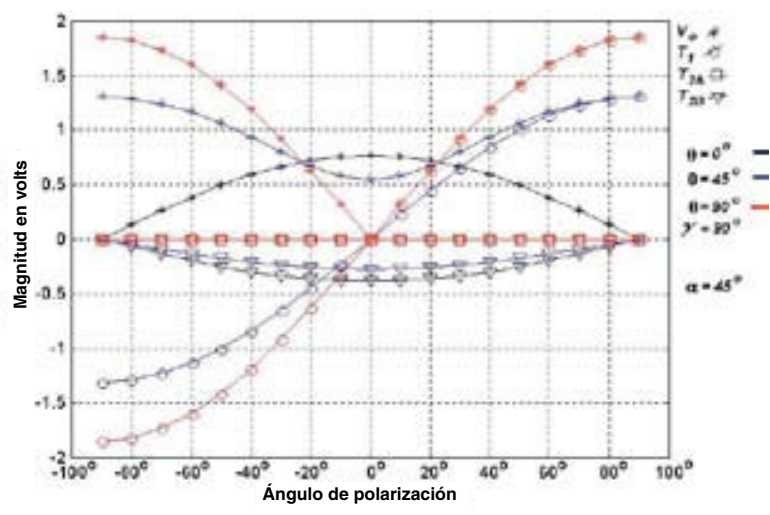

Figura 6a. Efectos del ángulo de polarización para diferentes ángulos de inclinación, considerando un corrimiento de fase de $90^{\circ}$ producido por el capacitor.

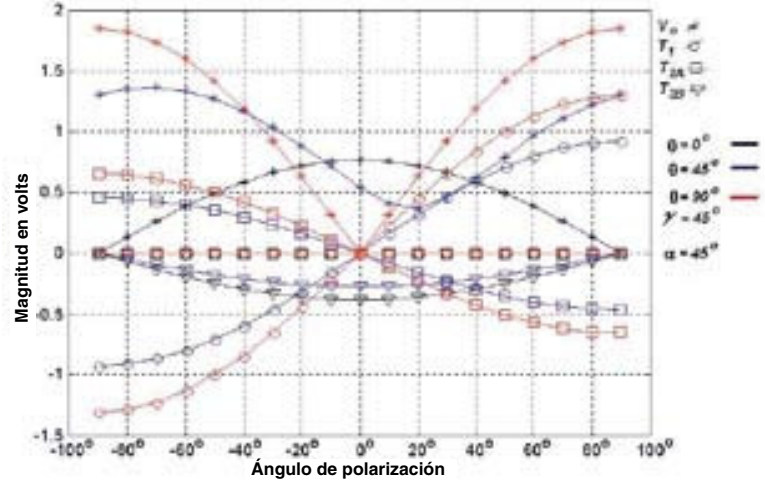

Figura 6b. Efectos del ángulo de polarización para diferentes ángulos de inclinación, considerando un corrimiento de fase de $45^{\circ}$ producido por el capacitor.

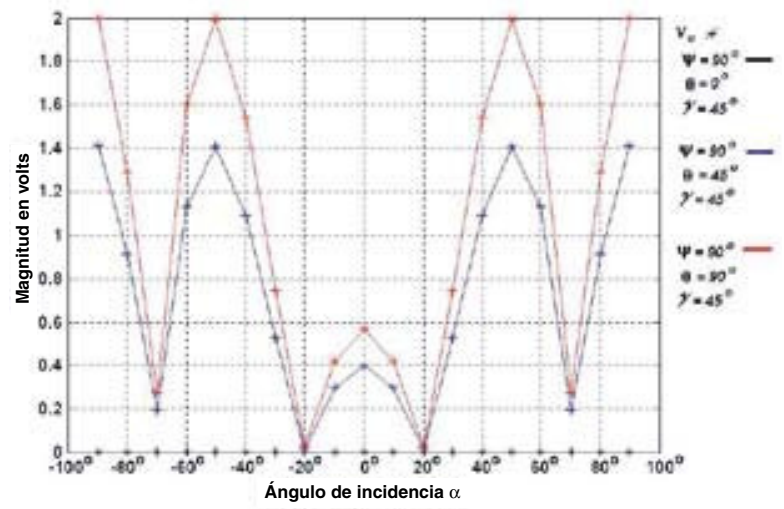

Figura 7a. Efectos del ángulo de incidencia de la señal para diferentes ángulos de inclinación y de polarización, considerando el corrimiento de fase constante.

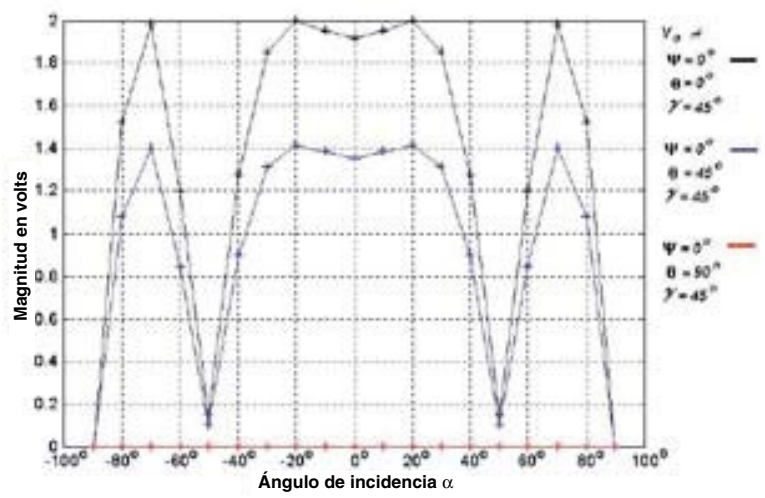

Figura 7b. Efectos del ángulo de incidencia de la señal para diferentes ángulos de inclinación y de polarización, considerando el corrimiento de fase constante. 
En las figuras anteriores se pueden observar los corrimientos que presenta la respuesta de la antena de dos elementos para las componentes del campo. Se tienen mejores condiciones, cuando la polarización y la inclinación es igual a $0^{\circ}$ como se puede observar en la figura 6 . El campo eléctrico tiene un comportamiento bastante estable, lo cual es normal porque está relacionado con el dipolo. Las variaciones más intensas se manifiestan en la componente de campo magnético, debido a que el capacitor está colocado en la antena de aro. Para ángulos de polarización y de inclinación igual a cero la componente dominante es la del campo eléctrico, la cual se puede identificar perfectamente en la figura $7 \mathrm{~b}$, en el intervalo del ángulo de incidencia de $\pm 45^{\circ}$. Los efectos del campo magnético se pueden identificar en la figura $7 \mathrm{a}$, donde se tiene un máximo para ángulos de incidencia de $+50^{\circ} \mathrm{y}-50^{\circ}$. Este comportamiento hace a la antena de dos elementos una herramienta potencial para el análisis de campos electromagnéticos en ambientes de multitrayectorias, conociendo los ángulos de incidencia de la señal recibida.

\section{PROCEDIMIENTO PARA LA DETERMINACIÓN DEL ÁNGULO DE INCIDENCIA}

De acuerdo con el concepto de trayectoria dominante, el voltaje de la señal de salida de la antena incluye varias trayectorias incidentes, las cuales no inciden con el mismo ángulo. Entonces, la envolvente de la señal resultante en un punto sobre el eje-a (figura 1) de la antena puede ser analizado por.

$$
V_{\text {out }}=\left|\sum_{i=1}^{n} A_{n} e^{j \varphi}\right|
$$

donde $A_{n}$ es una magnitud que incluye todos los efectos relacionados con la ganancia de la antena. $\varphi$ es la fase que incluye todos los efectos asociados con las diferentes longitudes de las trayectorias.

Si la antena se orienta con un ángulo de inclinación igual a cero y se supone que la polarización de la onda de campo eléctrico es de $45^{\circ}$, cuando la distancia $d$ entre la antena y un plano reflector tienen pequeñas variaciones (teoría de difracción de Fresnel), la ecuación (9) puede escribirse para describir una trayectoria espacial dominante con un comportamiento cuasiperiódico, esto es

$$
\begin{aligned}
V_{\text {out }}^{2} & =\sum_{i=1}^{n} A_{i}^{2}+\sum_{i=1}^{n-1} A_{1} A_{i+1} \cos \left(\varphi_{1}-\varphi_{i+1}\right) \\
& +2 \sum_{i=1}^{n-2} A_{2} A_{i+2} \cos \left(\varphi_{1}-\varphi_{i+1}\right) \\
& +\cdots+2 A_{n-1} A_{n} \cos \left(\varphi_{n-1}-\varphi_{n}\right)
\end{aligned}
$$

Ahora, aplicando la Transformada Discreta de Fourier (TDF) a la ec. (10), se pueden obtener las líneas espectrales relacionadas con las trayectorias incidentes en la antena. Como $\varphi=k_{o} d \cos \alpha$, donde $K_{o}=2 \pi / \lambda$ es la constante de propagación, $\lambda$ es la longitud de onda, $d$ es la distancia entre la antena y el plano reflector, y $\alpha$ es el ángulo de inclinación. La ec. (10) es función del ángulo de incidencia $(\alpha)$ de las trayectorias y de la distancia $(d)$. Analizando el voltaje de salida de la antena para tres trayectorias se tiene

$$
\begin{aligned}
V_{\text {out }}^{2}= & A_{1}^{2}+A_{2}^{2}+A_{3}^{2}+2 A_{1} A_{2} \cos \left[k_{o} d\left(\cos \alpha_{1}-\cos \alpha_{2}\right)\right] \\
& +2 A_{1} A_{3} \cos \left[k_{o} d\left(\cos \alpha_{1}-\cos \alpha_{3}\right)\right] \\
& +2 A_{2} A_{3} \cos \left[k_{o} d\left(\cos \alpha_{2}-\cos \alpha_{3}\right)\right]
\end{aligned}
$$

Aplicando la TDF a la ec. (11) se obtienen las líneas espectrales como función de $k_{o}$ que incluyen las características espaciales. Este espectro se deduce para características específicas, el cual puede ser usado como un patrón de referencia para determinar el ángulo de incidencia en función de la distancia y así obtener el tiempo de incidencia.

En la parte experimental, las mediciones pueden hacerse directamente en la antena (con el circuito acoplador) con un analizador de redes, o con un analizador de espectros, $o$ también con un osciloscopio que incluya la Transformada Rápida de Fourier. El espectro obtenido se puede comparar con el espectro de referencia que se obtiene por medio de la ec. (10) con parámetros preestablecidos de acuerdo con el escenario de propagación.

Por ejemplo, suponiendo tres diferentes casos con tres trayectorias se tiene (ver figura 8): 
1. Amplitudes de $A_{1}=1, A_{2}=0.8, A_{3}=0.4$, con ángulos de incidencia $\alpha_{1}=0^{\circ}, \alpha_{2}=30^{\circ}, \alpha_{3}=100^{\circ}$.

Línea espectral $S F_{o}=0$ tiene una amplitud $\mathbf{1 a}=(1)^{2}+(0.8)^{2}+(0.4)^{2}=1.8 V^{2}$

Línea espectral $S F_{1}=(1 / \lambda)\left(\cos 0^{\circ}-\cos 30^{\circ}\right)=0.134 / \lambda$ tiene una amplitud $\mathbf{1 b}=(2)(1)(0.8)=1.6 V^{2}$

Línea espectral $S F_{2}=(1 / \lambda)\left(\cos 0^{\circ}-\cos 120^{\circ}\right)=1.5 / \lambda$ tiene una amplitud $\mathbf{1 c}=(2)(1)(0.4)=0.8 V^{2}$

Líneaespectral $S F_{3}=(1 / \lambda)\left(\cos 30^{\circ}-\cos 100^{\circ}\right)=1.03 / \lambda$ tiene una amplitud $\mathbf{1 d}=(2)(0.8)(0.4)=0.64 \mathrm{~V}^{2}$

2. Amplitudes de $A_{1}=1, A_{2}=0.6, A_{3}=0.3$, con ángulos de incidencia $\alpha_{1}=0^{\circ}, \alpha_{2}=50^{\circ}, \alpha_{3}=120^{\circ}$.

Línea espectral $S F_{o}=0$ tiene una amplitud $\mathbf{2 a}=(1)^{2}+(0.6)^{2}+(0.3)^{2}=1.45 V^{2}$

Línea espectral $S F_{1}=(1 / \lambda)\left(\cos 0^{\circ}-\cos 50^{\circ}\right)=0.357 / \lambda$ tiene una amplitud $\mathbf{2 b}=(2)(1)(0.6)=1.2 V^{2}$

Línea espectral $S F_{2}=(1 / \lambda)\left(\cos 0^{\circ}-\cos 120^{\circ}\right)=1.5 / \lambda$ tiene una amplitud $\mathbf{2 c}=(2)(1)(0.3)=0.6 V^{2}$

Línea espectral $S F_{3}=(1 / \lambda)\left(\cos 50^{\circ}-\cos 120^{\circ}\right)=1.142 / \lambda$ tiene una amplitud $\mathbf{2 d}=(2)(0.6)(0.3)=0.36 V^{2}$

3. Amplitudes de $A_{1}=1, A_{2}=0.4, A_{3}=0.1$, con ángulos de incidencia $\alpha_{1}=0^{\circ}, \alpha_{2}=70^{\circ}, \alpha_{3}=150^{\circ}$.

Línea espectral $S F_{o}=0$ tiene una amplitud $\mathbf{3 a}=(1)^{2}+(0.4)^{2}+(0.1)^{2}=1.17 V^{2}$

Línea espectral $S F_{1}=(1 / \lambda)\left(\cos 0^{\circ}-\cos 70^{\circ}\right)=0.658 / \lambda$ tiene una amplitud $\mathbf{3 b}=(2)(1)(0.4)=0.8 V^{2}$

Línea espectral $S F_{2}=(1 / \lambda)\left(\cos 0^{\circ}-\cos 150^{\circ}\right)=1.866 / \lambda$ tiene una amplitud $\mathbf{3 c}=(2)(1)(0.1)=0.2 V^{2}$

Línea espectral $S F_{3}=(1 / \lambda)\left(\cos 70^{\circ}-\cos 150^{\circ}\right)=1.2 / \lambda$ tiene una amplitud $\mathbf{3 d}=(2)(0.4)(0.1)=0.08 \mathrm{~V}^{2}$

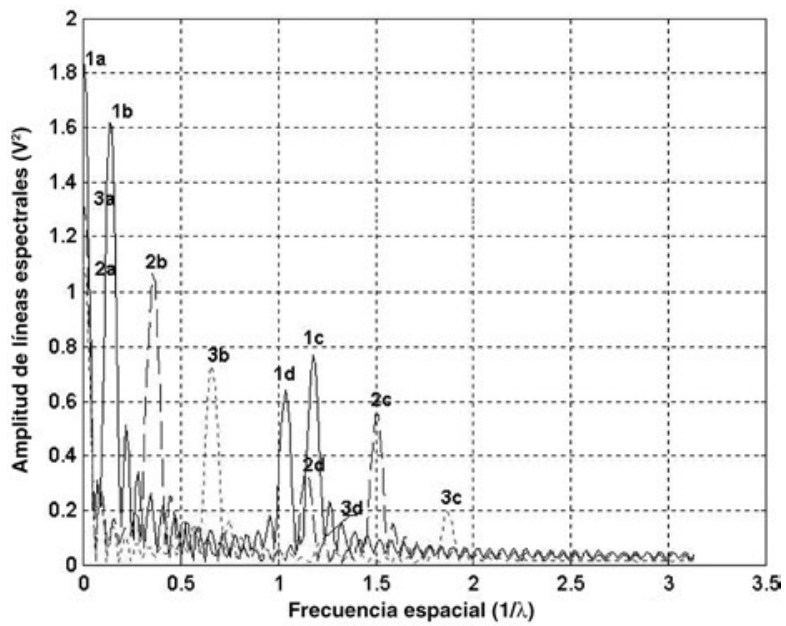

Figura 8. Espectro espacial.

Un patrón de referencia puede obtenerse para cantidades normalizadas, considerando que una de las ondas tiene un ángulo de incidencia igual a $0^{\circ}$. Para el caso de tres trayectorias el patrón de referencia puede construirse fácilmente y a partir de esos datos obtener un patrón con escala en ángulos. Para tres señales normalizadas $A_{1}=1 \mathrm{~V}^{2}, A_{2}=0.8 \mathrm{~V}^{2}, A_{3}=0.4 \mathrm{~V}^{2}$ con $\alpha=0^{\circ}$, $\alpha=45^{\circ}, \alpha=120^{\circ}$, respectivamente; se tiene la señal envolvente que se muestra en la figura 9 a y su espectro se muestra en la figura $9 \mathrm{~b}$. A partir de esos datos se produce el patrón que se muestra en la figura 10.

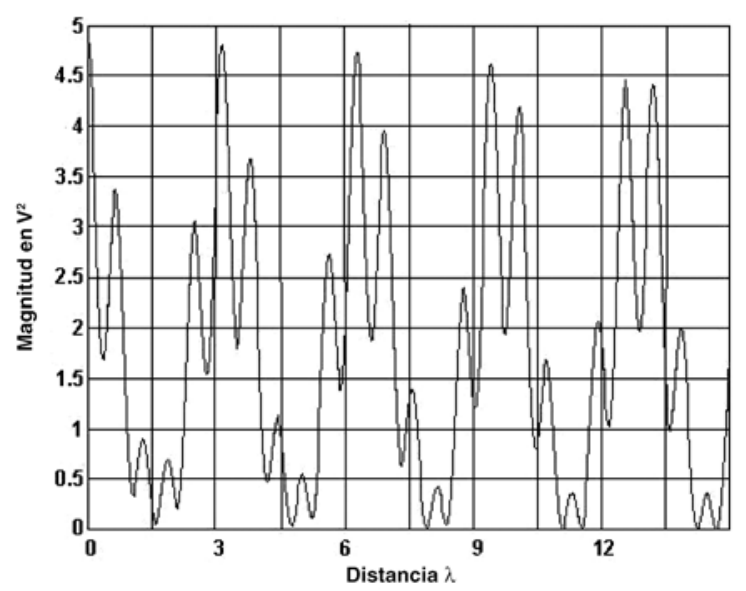

Figura 9a. Envolvente de señal con tres trayectorias vs. $\lambda$. 


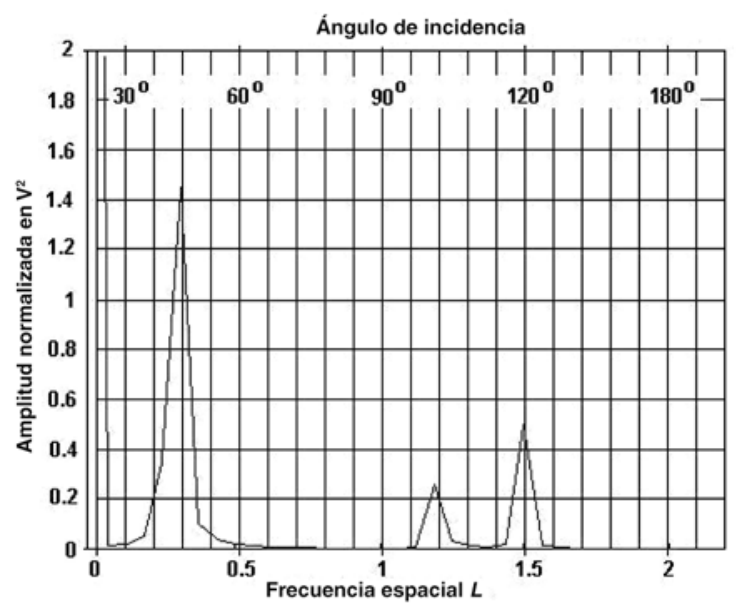

Figura 9b. Envolvente de señal con tres trayectorias vs. L.

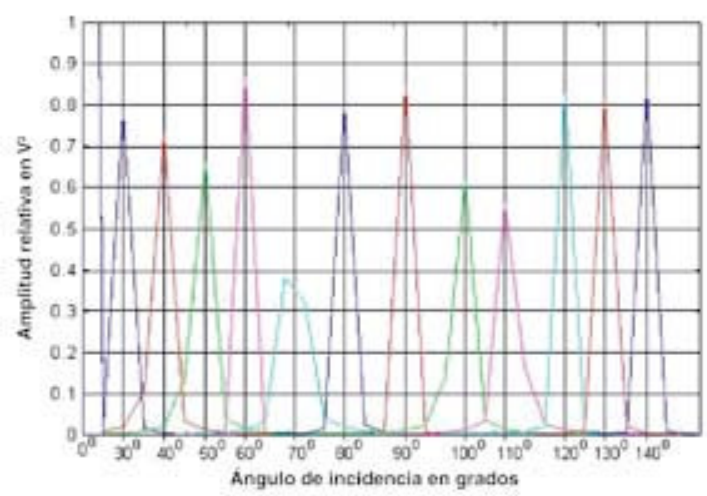

Figura 10. Patrón de referencia para la determinación del ángulo de incidencia.

\section{DIVERSIDAD ESPACIAL}

Como se ha mencionado, el ambiente electromagnético de los sistemas de comunicaciones inalámbricos se caracteriza por varios reflectores que producen desvanecimientos de multitrayectorias. Sin considerar las personas alrededor de la antena receptora se han medido desvanecimientos de señal hasta $30 \mathrm{~dB}$ [20]. Para minimizar estos desvanecimientos que se generan por diversos efectos, se han aplicado varias técnicas para aumentar la calidad de los sistemas de comunicaciones, sin recurrir a incrementos en la potencia de transmisión [17-19]. Una de esas técnicas es la diversidad espacial, que dentro de un ambiente de propagación de naturaleza aleatoria determina la señal independiente (no correlacionada) del proceso de información, por medio de un coeficiente de correlación. Este coeficiente se define como: el coeficiente de correlación espacial de una señal que llega a la antena receptora de acuerdo con el ángulo de incidencia y puede ayudar a determinar la distancia entre la antena y el plano reflector o la distancia entre dos antenas. De acuerdo con los análisis presentados anteriormente, aplicando los conceptos de trayectoria dominante y el patrón de interferencia dominante, esta técnica permite analizar los efectos de profundidad de los nulos y optimizar desvanecimientos. El coeficiente de correlación de frecuencia espacial se determina por:

$$
\rho_{\text {des }}=\frac{\left\langle\left(\left|V_{o 1}\right|-\left\langle\left|V_{o 1}\right|\right\rangle\right)\left(\left|V_{o 2}\right|-\left\langle\left|V_{o 2}\right|\right\rangle\right)\right\rangle}{\sigma_{o 1} \sigma_{o 2}}
$$

donde $\left|V_{o 1}\right|$ es la magnitud de la primera trayectoria dominante, $\left|V_{o 2}\right|$ es la magnitud de la segunda trayectoria dominante y $\sigma$ corresponde a la desviación estándar de cada una de las trayectorias. Cada magnitud se describe por una función de distribución acumulativa.

Este coeficiente también puede obtenerse por medio de los ángulos de incidencia y se determina por:

$$
\rho_{a i}=\frac{\left\langle\left(\left|\alpha_{o 1}\right|-\left\langle\left|\alpha_{o 1}\right|\right\rangle\right)\left(\left|\alpha_{o 2}\right|-\left\langle\left|\alpha_{o 2}\right|\right\rangle\right)\right\rangle}{\sigma_{o 1} \sigma_{o 2}}
$$

donde $\left|\alpha_{o 1}\right|$ es el ángulo de la primera trayectoria dominante, $\alpha_{o 2} \mid$ es el ángulo de incidencia de la segunda trayectoria dominante y $\sigma$ es la desviación estándar de los ángulos correspondientes a cada trayectoria. Desde luego, los ángulos se deben de describir con una distribución acumulativa

\section{EXPERIMENTACIÓN Y VALIDACIÓN DE LOS MODELOS}

La parte experimental se realizó en un área abierta sobre asfalto (conductividad igual a cero y constante dieléctrica 2.68), teniendo como fuente un dipolo. La distancia entre la antena transmisora y la receptora (antena de dos elementos) es de $6 \mathrm{~m}$, en la cual se optimizó la comunicación a $475 \mathrm{MHz}$, para no tener interferencias con sistemas de comunicación comercial. Como plano reflector se utilizó una lámina de aluminio de $3.40 \mathrm{~m}$ de largo y $1.20 \mathrm{~m}$ de ancho y se fijó verticalmente en la parte trasera de la antena receptora sobre una estructura de madera que permite alinear la antena al centro y moverla horizontalmente hasta $1.5 \mathrm{~m}$. La distancia de las antenas al asfalto se fijó a $0.16 \mathrm{~m}$ que corresponde a $1 / 4 \lambda$, esta distancia se optimizó de acuerdo a la teoría de difracción del Fresnel para mínimos efectos de la reflexión del plano horizontal. Las 
mediciones se realizaron con un analizador de espectros para ángulos de incidencia $\alpha=-\pi / 2$ a $+\pi / 2$, ángulo de inclinación $\theta=0^{\circ}$, ángulos de polarización de la señal $\Psi=-\pi$ a $+\pi$, y distancia del plano receptor a la antena $d=0.1 \mathrm{~m}$ a $0.75 \mathrm{~m}$. Se realizaron 10.000 mediciones y se construyó un histograma con 25 barras de $-10 \mathrm{~dB}$ de ancho. La gráfica de la función de distribución acumulativa se muestra en la figura 11. Para la distribución espacial se tomó como referencia el ángulo de inclinación $\alpha=0^{\circ}$ y se identificaron los tres niveles de mayor frecuencia de incidencia $\left(A_{1}=-8.6 d B, A_{2}=-13.4 d B, A_{3}=-16.2 d B\right)$. La gráfica de la envolvente y del patrón espacial dominante se muestra en la figura 12a y su espectro en la figura $12 \mathrm{~b}$. Con los datos obtenidos los coeficientes de correlación son: $\rho_{\text {des }}=0.93$ y $\rho_{a i}=0.81$ para una distancia de $0.53 \mathrm{~m}$ $(0.83 \lambda)$ tomando como referencia la señal sin reflector.

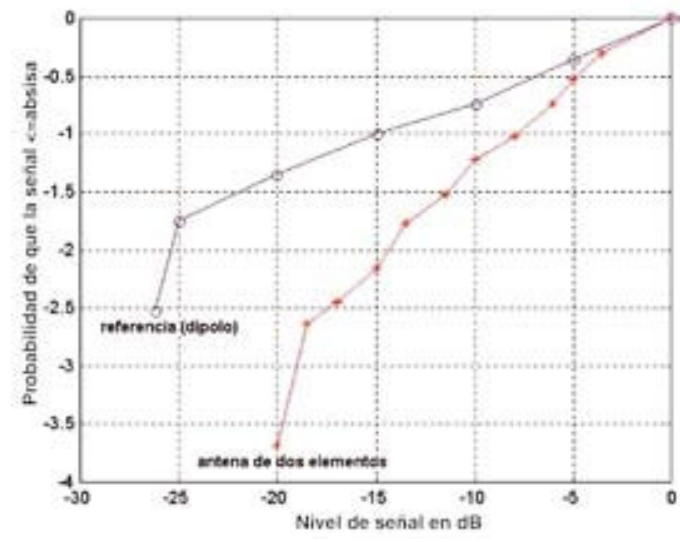

Figura 11. Función de distribución acumulativa.

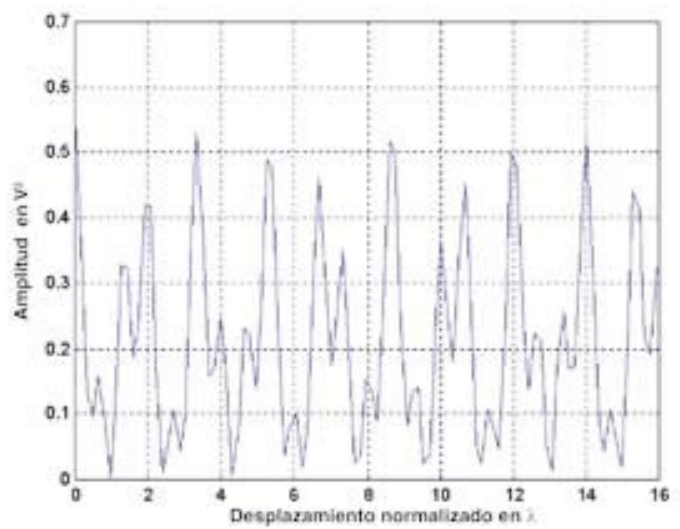

Figura 12a. Envolvente y espectro espacial de la señal recibida de acuerdo al concepto de patrón espacial de interferencias dominantes.

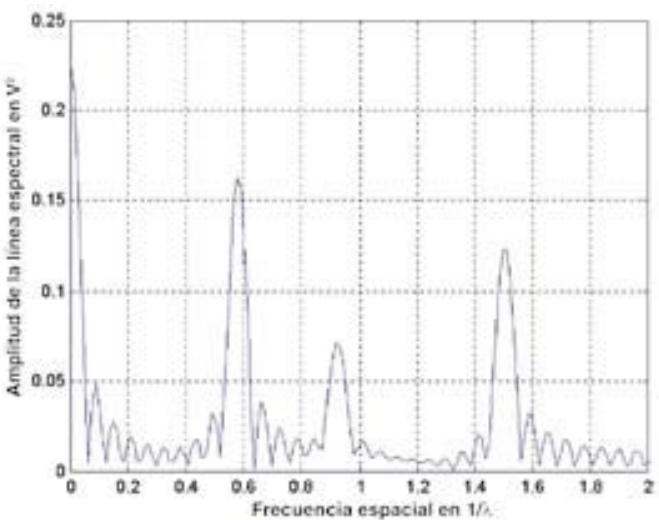

Figura 12b. Envolvente y espectro espacial de la señal recibida de acuerdo al concepto de patrón espacial de interferencias dominantes.

\section{CONCLUSIONES}

La determinación de la diversidad espacial es de utilidad en los sistemas de comunicaciones inalámbricos, como por ejemplo: permite analizar la eficiencia de la separación de las antenas cuando se tienen arreglos, o también determinar la directividad en los enlaces de microondas y como aplicación integrada a la localización de sistemas móviles. El procedimiento que se reporta es bastante simple y no requiere de sistemas de adquisición de datos complicados y para integrarse a sistemas sólo se requiere un DSP con la Trasformada Rápida de Fourier. Este procedimiento utilizado con la antena de dos elementos permite estimar las componentes del campo electromagnético, ya que orientada en una determinada dirección, uno de los componentes del campo es dominante. Esta última aplicación es importante en el análisis del ambiente electromagnético de multitrayectorias en zonas urbanas, tanto en espacios abiertos como cerrados, debido a que los obstáculos que producen la reflexión, difracción o dispersión de las ondas electromagnéticas no son conductores perfectos y los componentes del campo sufren diferentes niveles de atenuaciones, entonces con este procedimiento para ciertas zonas se pueden buscar las condiciones óptimas de recepción.

\section{REFERENCIAS}

[1] R.T. Comptom. "An adaptive Array in a SpreadSpectrum Communication System". Proc. of the IEEE. Vol. 66 N 3. March 1978.

[2] R.G. Vaughan and J.B. Andersen. "Antennas diversity in mobile communications". IEEE, Trans. 
Veh. Technol. Vol. VT-36, pp. 149-172. November 1987.

[3] L.C. Godara. "Application of Antennas Array to Mobile Communications, Part I: Performance improvement, Feasibility, and Systems Consideration". Proc. of the IEEE. July 1997.

[4] L.C. Godara. "Application of Antennas Array to Mobile Communications, Part II: Beamforming and Direction of Arrival Consideration". Proc. of the IEEE. August 1997.

[5] W.C.Y. Lee. "Effects of correlation between two mobile radio base station antennas". IEEE Trans. Commmun. Vol. COM-21 No 11, pp. 1214-1224. 1973.

[6] G.E. Carazza, V. Degli-Esposti, M. Frullone and G. Riva. "A characterization of indoor space and frequency diversity by ray-tracing modeling". IEEE L. Select. Areas Comm. Vol. 14 No 3, pp. 411-419. 1996.

[7] J.H. Tarng, Shan Chang, Jiunn-Ming Huang and Yih-Min Tu. "A New Efficient Hybrid Model for Estimate Space Diversity in Indoor Environment". IEEE Trans. Veh. Technol. Vol. $49 \mathrm{~N}^{\circ}$ 2, pp. 457466. March 2000.

[8] R.B. Ertel, P. Cardieri, K.W. Sowerby, T.S. Rappaport and J.H. Reed. "Overview of spatial channel models for antenna array communication systems". IEEE Personal Communication, pp. 10-22. February 1998.

[9] J.C. Liberti and T.S. Rappaport. "Smart Antennas for Wireless Communications:IS-95 and Third Generation CDMA Application". Prentice Hall. NJ. 1999.

[10] R.G. Vaughn. "Polarization diversity in mobile communications". IEEE Trans. Veh. Tech. Vol VT-39 No 3, pp. 177-186. August 1990.

[11] M.A. Jensen and Y. Rahmat-Samii. "Characterization of electromagnetically coupled superquadratic loop antennas for mobile communications applications". IEE Proc. H, Vol. 141, pp. 85-93. April 1994.

[12] M. Muramoto, K Itoh, N. Ishii and K. Sasaki. "A proposal of a small planar loop antenna whose polarization direction can be switched electrically". IEEE AP- Int. Symp. Baltimore, MD. Vol. 1, pp. 726-729. July 1996.

[13] M. Muramoto, N. Ishii and K. Itoh. "Characteristic of a small planar loop antenna". IEEE Trans. Antennas Propagat. Vol. 45 No 12, pp. 1818-1821. December 1997.

[14] J. Richter and M.O. Al-Nuaimi. "Resolution of constituent components in multipath field using DFT". Electronics Letters. Vol. $31 \mathrm{~N}^{\mathrm{o}} 17$, pp. 14151416. $17^{\text {th }}$ August 1995.

[15] J. Richter and M.O. Al-Nuaimi. "Procedure for the special resolution of multipath signals in urban radio links". IEE Proc. Microwave and Antenna Propagation. Vol. $147 \mathrm{~N}^{\circ} 3$, pp. 224-230. June 2000.

[16] K.H. Sayidmarie and A.M. Abbosh. "Holographic prediction of ground multipath parameters from range gain patterns". IEE Proc. Microwave and Antenna Propagation. Vol. 140 No 5, pp. 367-372. 1993.

[17] W.F. Gabriel. "Spectral analysis and adaptive array superresolution technique". Proc. IEEE. Vol. 68 No 6, pp. 654-666. 1980.

[18] R.J.L. Bultitude. "Measurements, characterization and modeling of indoor $800 / 900 \mathrm{MHz}$ radio channel for digital communications". IEEE Communication Magazine. Vol. 25 N$^{\circ}$ 6. June 1987.

[19] E.N. Gilbert. "Energy reception for mobile radio". Bell. Sist. Tech. J., Vol. 4 No $^{\circ}$, pp. 1779-1803. 1965.

[20] R.H. Clere. "A Statistical Theory of Mobile Radio reception”. Bell. Sist.. Tech. J. Vol. 47, pp. 9571000. 1968. 